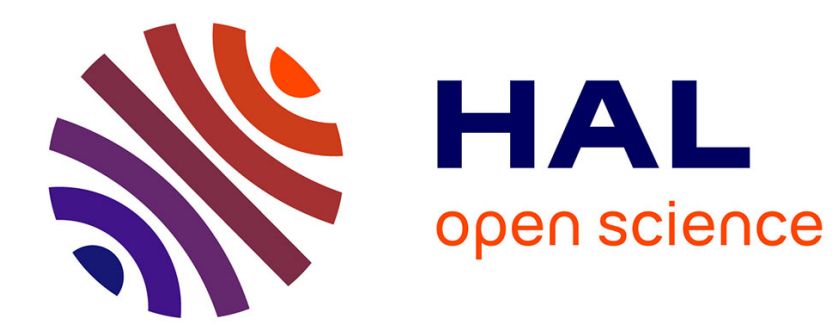

\title{
ANGLE RESOLVED PHOTOEMISSION FROM CLEAN SURFACES
}

\author{
Y. Petroff
}

\section{To cite this version:}

Y. Petroff. ANGLE RESOLVED PHOTOEMISSION FROM CLEAN SURFACES. Journal de Physique Colloques, 1978, 39 (C4), pp.C4-149-C4-160. 10.1051/jphyscol:1978419 . jpa-00217453

\section{HAL Id: jpa-00217453 https://hal.science/jpa-00217453}

Submitted on 1 Jan 1978

HAL is a multi-disciplinary open access archive for the deposit and dissemination of scientific research documents, whether they are published or not. The documents may come from teaching and research institutions in France or abroad, or from public or private research centers.
L'archive ouverte pluridisciplinaire HAL, est destinée au dépôt et à la diffusion de documents scientifiques de niveau recherche, publiés ou non, émanant des établissements d'enseignement et de recherche français ou étrangers, des laboratoires publics ou privés. 


\title{
ANGLE RESOLVED PHOTOEMISSION FROM CLEAN SURFACES
}

\author{
Y. PETROFF \\ L.U.R.E., Université Paris-Sud, Bât. 209 C, 91405 Orsay Cedex, France
}

\begin{abstract}
Résumé. - Pendant longtemps les méthodes optiques ont été les seules employées pour obtenir des informations sur la structure de bandes.

La photoémission angulaire qui est une amélioration d'une vieille technique est en train de devenir l'outil le plus puissant pour l'étude des niveaux électroniques.

Nous ne parlerons ici que des états de volume. Des exemples sont donnés pour trois cas types :

1) Les matériaux lamellaires pour lesquels il est possible d'obtenir la dispersion des bandes d'énergie d'une manière simple à cause de la faible dispersion le long de $k_{z}$.

2) Les semiconđucteurs à trois dimensions tels que $\mathrm{PbS}$ et $\mathrm{PbSe}$ pour lesquels un modèle de densité d'états à une dimension permet d'expliquer les résultats expérimentaux et d'obtenir la dispersion le long de directions de haute symétrie.

3) Les métaux ( $\mathrm{Cu}$ et $\mathrm{Ni}$ ) : les résultats du cuivre, plus simple à cause de sa bande $\mathrm{d}$ complètement pleine, sont comparés à des calculs employant le formalisme de la diffraction d'électrons lents (DEL). Le problème des effets de corrélations est discuté brièvement. Enfin dans le dernier paragraphe nous rapportons la première observation d'un effet de résonance en photoémission.
\end{abstract}

\footnotetext{
Abstract. - For a long time the informations about the band structure of solids were obtained only by optical measurements. Angle resolved photoelectron spectroscopy (ARPES) which is a sophisticated refinement of an old technique has become a very powerful tool to get informations on the electronic levels.

We limit our discussion to volume states. Examples are given for three cases :

1) Layers materials for which it is possible to map the bands in a very simple way due to the flatness of the bands along $k_{z}$.

2) Three dimensional semiconductors as $\mathrm{PbS}$ and $\mathrm{PbSe}$. For these an one dimensional density of states model explains very well the experimental data and allows also to obtain the dispersion along high symmetry lines.

3) Metals as $\mathrm{Cu}$ and $\mathrm{Ni}$ : in this case comparison is done with calculations using the L.E.E.D. formalism. The problem of correlation effects is shortly discussed. Finally a strong resonance effect observed for the first time in photoemission will be reported.
}

1. Introduction. - The aim of this talk is to present an overview of the possibilities of the angle resolved photoemission technique for the study of the electronic levels of solids.

I will not discuss at all the case of surfaces states or adsorbates : this part will be covered by N. Smith. Also for the volume states I will limit myself to energy distribution experiments and leave the constant final (CFS) and initial (CIS) energy spectra to G. Lapeyre, who will discușs also the polarization dependent photoemission.

Since the Hamburg Conference the activity in the area of angular resolved photoemission spectroscopy (ARPES) has been growing up very fast and it is impossible to cover all the field. I have been forced to choose few experiments and I apologize in advance for that. The principle of (ARPES) is quite simple; let denote by $\theta$ the polar angle (angle between the analyzer and the normal to the surface) and by $\varphi$ the azimuthal angle (see Fig. 1) and collect only the electrons emitted in a small solid angle. In that case we measure not only the energy of the photoelectrons but also their momentum $\mathbf{K}$. The problem is to relate $\mathbf{K}$ to the wave vector of the electron in the solid $\mathbf{k}$. We will suppose that there is conservation of the component parallel to the surface [1]

$$
\mathbf{K}_{\|}=\mathbf{k}_{\|}+\mathbf{G}_{\|}
$$

where $\mathbf{G}_{\| \mid}$is the parallel component of any reciprocal lattice vector $\mathbf{G}$.

If $E_{\mathrm{c}}$ is the kinetic energy of the photoelectron

$$
k_{\|}=\left[\frac{2 m E_{\mathrm{c}}}{\hbar^{2}}\right]^{1 / 2} \sin \theta
$$

we have to notice that $k_{z}=k_{\perp}$, the component of $\mathbf{k}$ perpendicular to the surface does not appear in the equation above and it is indeterminate : [2] this is the 


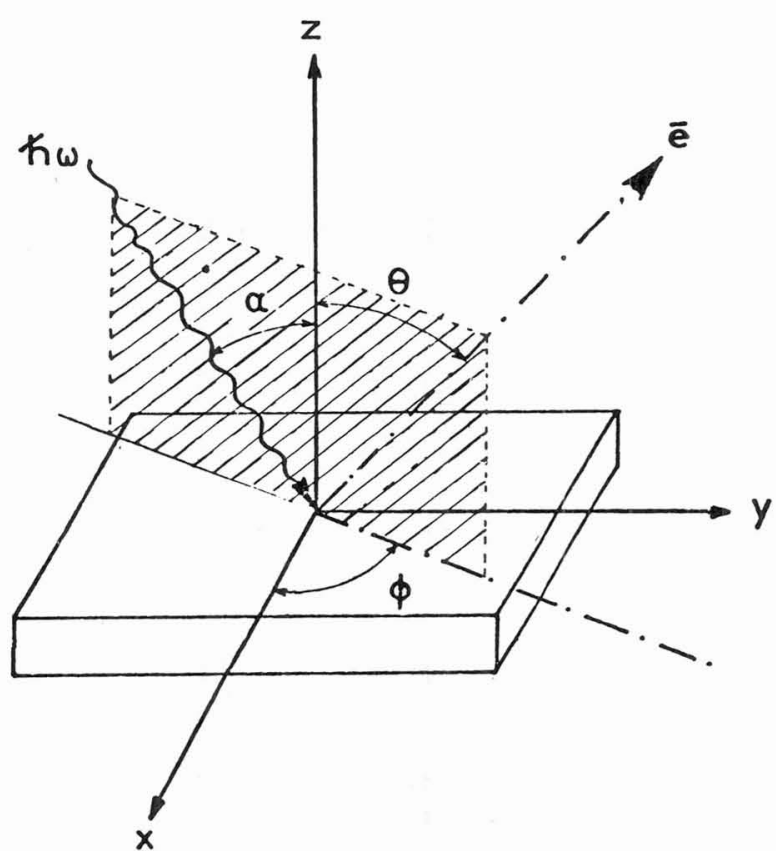

FIG. 1. - Angles used in ARPES experiments; $\alpha$ is the angle between the photon beam and the normal to the surface.

main difficulty. So the first attempt was to study special cases :

- Layer compounds where the dispersion along $k_{\perp}$ is small in first approximation.

- Normal emission : by collecting only electrons emitted at the normal $\left(\theta=0, k_{\|}=0\right)$ we can obtain the dispersion along $k_{\perp}$ by varying the photon energy $\hbar \omega$.

In section 3, I discuss the case of layers compounds. Section 4 is devoted to three dimensional semiconductors and we will show that the dream of determining the three dimensional band structure of a solid directly from experiment can be reached...

In section 5 we will review $\mathrm{Cu}$ and $\mathrm{Ni}$ which are quite interesting due to the difference in their $\mathrm{d}$ band and we will show that a sophisticated calculation using the L.E.E.D. formalism gives a remarquable agreement with experiment. Finally we will report on a strong resonance effect observed on nickel (100).

2. Experimental set-up. - Some of the experiments that I will discuss now were performed at the Orsay (ACO) storage ring : so I will describe shortly the system that we use there. It is quite similar to most of the photoemission set-up excepted that we have the chance to replace the He discharge lamp by the light emitted by the storage ring.

The following configuration (Fig. 2) is used : $5 \mathrm{mrad}$. of the light emitted by the storage ring are focused at the entrance slit of the monochromator by a toroidal mirror. The size of the point source $S(\varnothing=1 \mathrm{~mm})$ is reduced by a factor 3 . The monochromator (JobinYvon LHT 30) is based on a simple [3] rotation of a toroidal holographic grating and gives a resolution

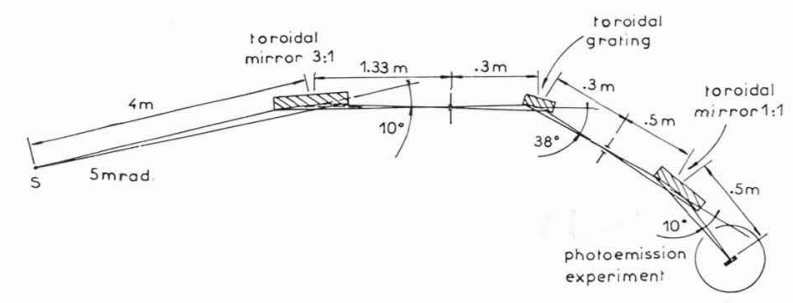

FIG. 2. - Experimental set up; $\mathrm{S}$ is the point source in the storage ring.

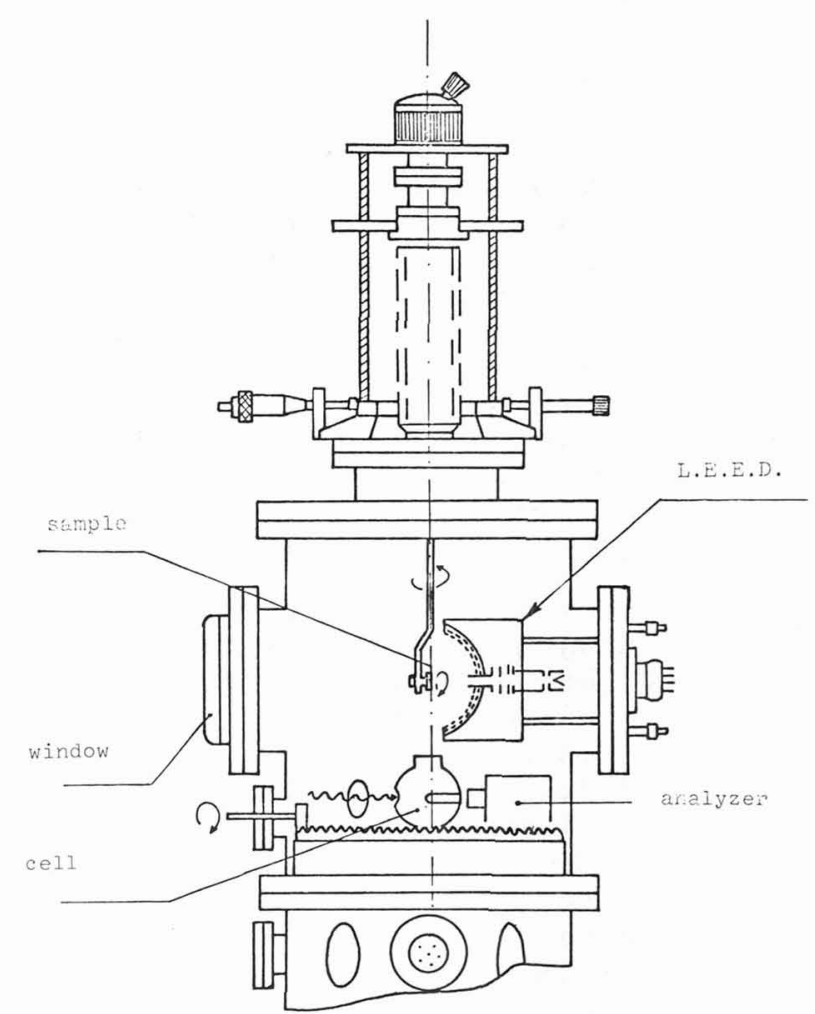

FIG. 3. - Experimental chamber; notice the level 1 where the surface can be caracterized by Auger and L.E.E.D. and the level 2 where the analyzer can be rotated in the horizontal plane.

of the order of $1 \AA$. At the exit the photon beam is focused to a size $<0.5 \times 0.7 \mathrm{~mm}^{2}$. The experimental chamber is represented on figure 3 . At the first level the sample can be cleaved or ion bombarded and caracterized by L.E.E.D. and Auger spectroscopies. At the second the energy analyzer, which is a $127^{\circ}$ cylindrical type, is rotated around the sample. The acceptance angle is $1^{\circ} \times 2^{\circ}$ and it was operated at an energy resolution varying between 100 and $300 \mathrm{meV}$.

3. Layers compounds. - We look at shortly the properties of layer compounds which have made them attractive for angle-resolved photoemission experiments. As an example we have chosen GaSe which has been studied by different groups [4], [5], [6], [7] but I would like to mention the pioneering work of Smith and Traum on $\mathrm{TaS}_{2}$ and $\mathrm{TaSe}_{2}[8]$. 
GaSe is a layered compound with a hexagonal structure; it consists of thin tightly bound layers. Each of these has four close-packed mono-atomic sheets (Se-Ga-Ga-Se).

The crystal structure is shown on figure 4 . The stacking sequence of the sandwiches gives rise to a lot of crystal modifications (polytypes).

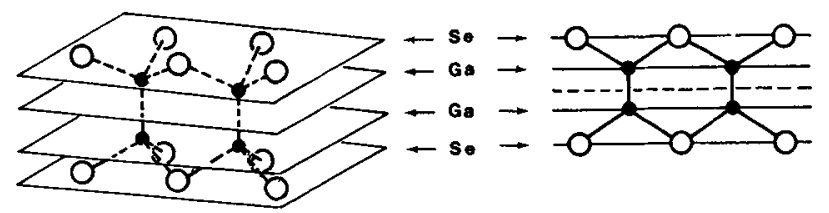

FIG. 4. - Crystal structure of GaSe $(\beta)$.

The interaction between adjacent layers is of the Van Der Waals type.

The Brillouin zone (Fig. 5) is hexagonal and the important symmetry directions are $\Gamma \mathrm{A}, \Gamma \mathrm{M}$ and $\Gamma \mathrm{K}$.

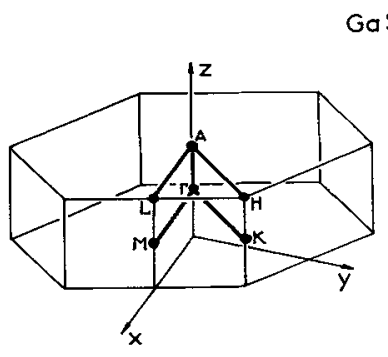

a)

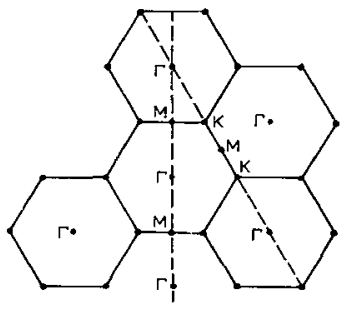

b)
FIG. 5. - Three (a) and two $(b)$ dimensional Brillouin zone of GaSe.

In first approximation we suppose that the electrons are confined in the sandwiches which means that the dispersion along $\Gamma$ A (perpendicularly to the plane of the layers) is small.

We suppose that the component of the wave vector parallel to the surfaces $k_{\|}$, is conserved during the emission process : in this case the relation between the kinetic energy $E_{\mathrm{c}}$ of the electron, the polar angle $\theta$ and $k_{\|}$is given by equation (2).

If $E_{\mathrm{val}}$ is the energy of the initial state

$$
E_{\mathrm{val}}=E_{\mathrm{c}}-\hbar \omega+W
$$

where $W$ is the energy difference between the vacuum level and the top of the valence band.

By combining (3) and (2)

$$
E_{\mathrm{val}}=\left[\frac{\hbar^{2}}{2 m} \sin ^{2} \theta\right] k_{\|}^{2}-(\hbar \omega-W)
$$

we have two possibilities to obtain $E_{\mathrm{val}}\left(\mathbf{k}_{\|}\right)$:

- choose one $\hbar \omega$ and vary $\theta$,

- vary $\hbar \omega$ for a fixed $\theta$.
We will consider the first approach where the final state is the same for any $\theta$. The figure 6 shows the photoelectron energy spectra along the azimuth $\Gamma \mathrm{M} \Gamma$ and $\Gamma$ KM obtained for a photon energy $\hbar \omega=27 \mathrm{eV}$. The background due to the secondary electrons has been substracted for clarity but has no influence on the structures.

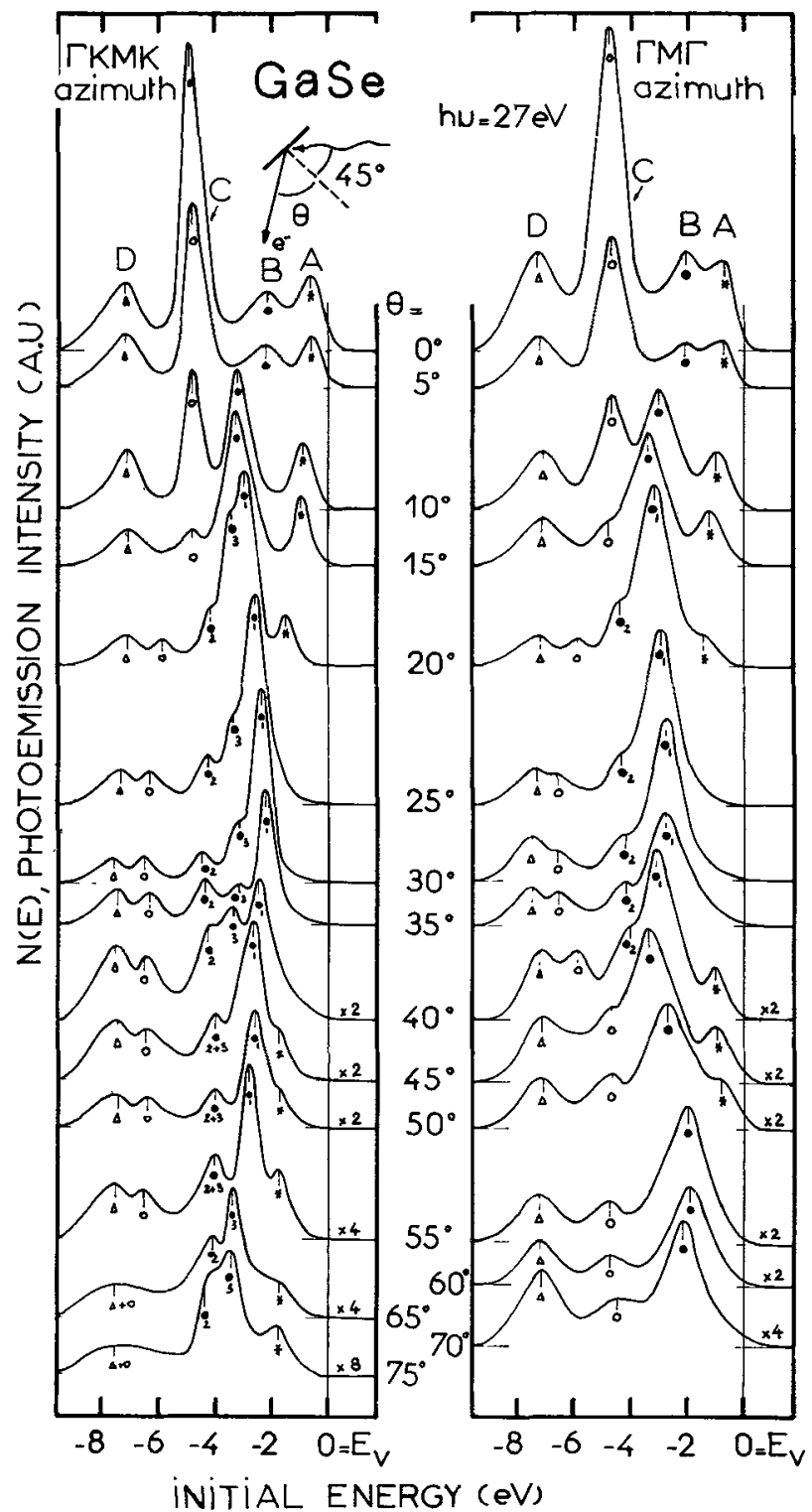

FIG. 6. - Energy distribution curves of the photoelectrons in function of the polar angle, obtained at $\hbar \omega=27 \mathrm{eV}$ along $\Gamma \mathrm{KMK}$ and $\Gamma \mathrm{M} \Gamma$ azımuths. A, B, C and D correspond to the groups of bands discussed in the text.

The valence band of GaSe has five groups of bands that we label (from the top of the valence band) A, B, C, D and E corresponding to two different bonds $\mathrm{Ga}-\mathrm{Ga}$ and $\mathrm{Ga}-\mathrm{Se}$ (if we neglect the weak Van der Waals interaction between layers). The gallium and selenium $4 \mathrm{~s}$ and $4 \mathrm{p}$ atomic like orbitals are involved in these bands. 
- The group A which corresponds to the uppermost valence band has a bonding character between the $\mathrm{Ga}$ atoms and a pronounced $\mathrm{p}_{z}$ like charge [9] is found around the $\mathrm{Se}$ atoms. This has been confirmed recently by polarization dependent photoemission experiments [10] showing that the peak corresponding to A disappears for s-polarized light.

- B is due to $\mathrm{p}_{x}, \mathrm{p}_{y}$ orbitals in the Ga-Se bond (mostly covalent).

- The next two structures C and D correspond mainly to $s(\mathrm{Ga})$ electrons. These states hybridize by adding $\mathrm{p}_{z}$ character and form a pair of bonding (D) and antibonding $(\mathrm{C})$ bands of the $\mathrm{Ga}-\mathrm{Ga}$ bond.

$-E$ takes its origin from the $4 \mathrm{~s}$ levels of Se. At low photon energy $(\hbar \omega<50 \mathrm{eV})$ we have not been able to observe this band which is seen by X.P. S. [1 1$]$ and also at $\hbar \omega=85 \mathrm{eV}[12]$.

We ignore the change in intensity of the different peaks and we follow the change in the positions when $\theta$ varies between 0 and $75^{\circ}$. The results are given in figure $7 a$ for the $\Gamma \mathrm{M} \Gamma$ azimuth and $7 b$ for the $\Gamma \mathrm{KMK}$. As seen by looking at the two dimensional Brillouin zone (Fig. 5) for a value of $\theta$ larger than $25^{\circ}$ we move in the second zone. We expect to have a mirror symmetry about the $M$ point and this is exactly verified. The figure $7 c$ represents the bands in the reduced zone scheme : the dashed part (Fig. 8)

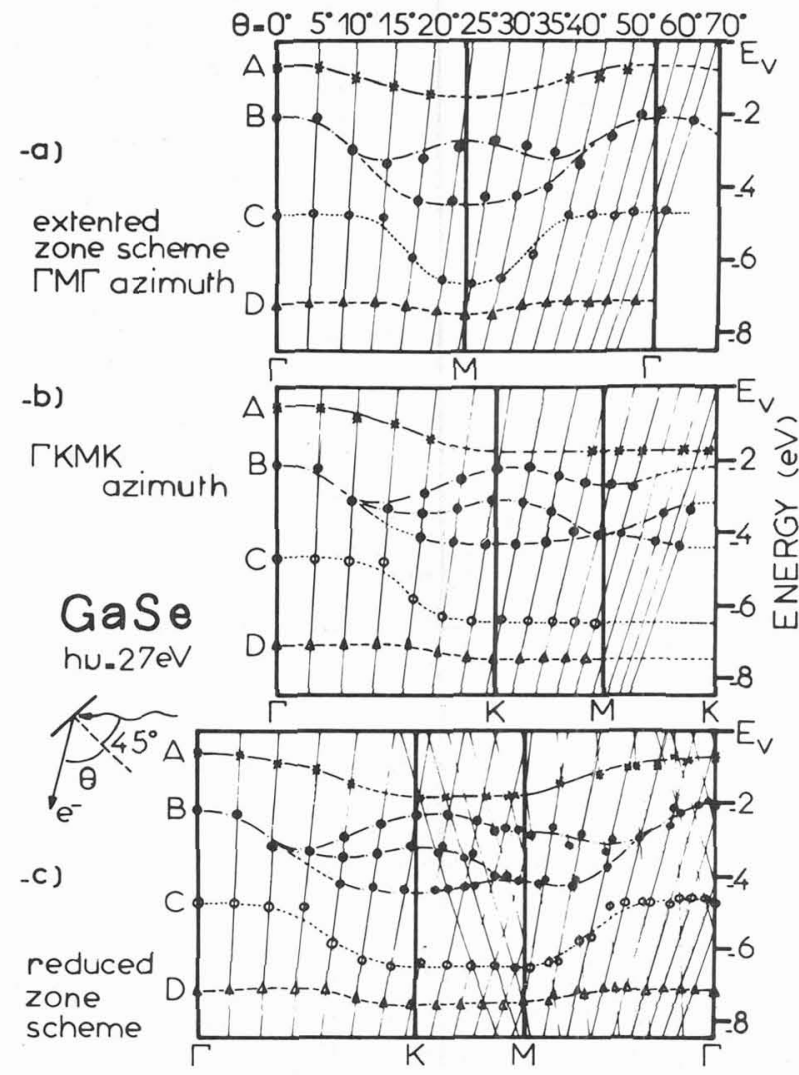

Fig. 7. - a) Experimental band structure obtained at $\hbar \omega=27 \mathrm{eV}$ along the $\Gamma \mathrm{M} \Gamma$ azimuth. Notice the mirror symmetry around $\mathrm{M}$. b) Experimental band structure obtained at $\hbar \omega=27 \mathrm{eV}$ along the $\Gamma \mathrm{KMK}$ azimuth. $c$ ) Experimental band structure in the reduced zone scheme.

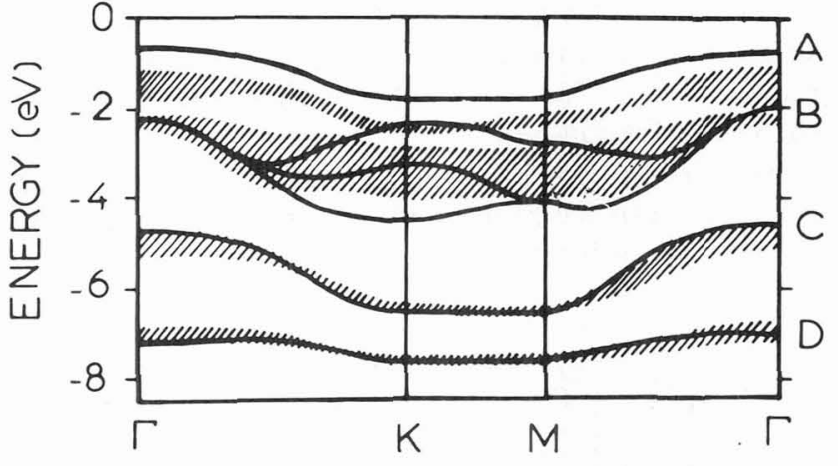

FIG. 8. - Comparison of the Schlüter's pseudopotential calculation (dashed part), shifted by $1.2 \mathrm{eV}$ and the experimentally determined energy bands. /II///, theoretical band structure shifted by $1.2 \mathrm{eV}$. —_ experimental band structure.

corresponds to the pseudopotential band structure of Schlüter [13] et al. There is an overall agreement if we shift the calculation by about $1 \mathrm{eV}$. There are some discrepancies concerning the bandwidth of the group B.

We have performed measurements also at $\hbar \omega=33,41$ and $45 \mathrm{eV}$ and found that the dispersion of the bands is almost independant of the final state [14].

Very similar results have been obtained by Larsen et al. [15] for InSe (see Fig. 11) by Grandke and Ley for GeS [16].

Normal Emission :

It is easy $[6,17]$ to test the assumption that in the layer materials the bands have a small dispersion along $k_{\perp}$. If we work at the normal $\left(\theta=0\right.$ and $\left.k_{\|}=0\right)$ and we suppose free electron like conduction bands

$$
\frac{\hbar^{2} k_{\perp}^{2}}{2 m}=E_{\mathrm{c}}+W_{0}
$$

where $W_{0}$ is the inner potential.

Then by measuring the photoemission distribution curves at $\theta=0$ for various $\hbar \omega$ we obtain the dispersion along $k_{\perp}(\Gamma \mathrm{A})$. This has been done [16] for photon energies between 21 and $50 \mathrm{eV}$ (Fig. 9). Using the equation (4), choosing arbitrarly the origin of $W_{0}$ and projecting the results in the first Brillouin zone we have obtained the dispersion along $\Gamma \mathrm{A}$ (Fig. 10a). We see that the bands $\mathrm{B}$ and $\mathrm{D}$ have almost no dispersion $(\leqslant 300 \mathrm{meV})$. The experimental points of bands $\mathrm{A}$ and $\mathrm{C}$ show a strong variation in energy along $\Gamma \mathrm{A}$. Since from the theoretical calculation [13] we expect two bands which are degenerate at $\Gamma$, contributing to each of these peaks, we take the energy variation of the experimental points as a strong evidence of dispersion although the points do not seem to align along distinct bands. Such a behaviour is expected for peak A from Schlüter's calculation of the electron charge density. $\mathrm{He}$ has found a pronounced $\mathrm{p}_{z}$ charge around the Se atoms extending towards the neighbouring layers; then we expect a three dimensional character. The comparison with the Schlüter's calculation along $\Gamma \mathrm{A}$ 


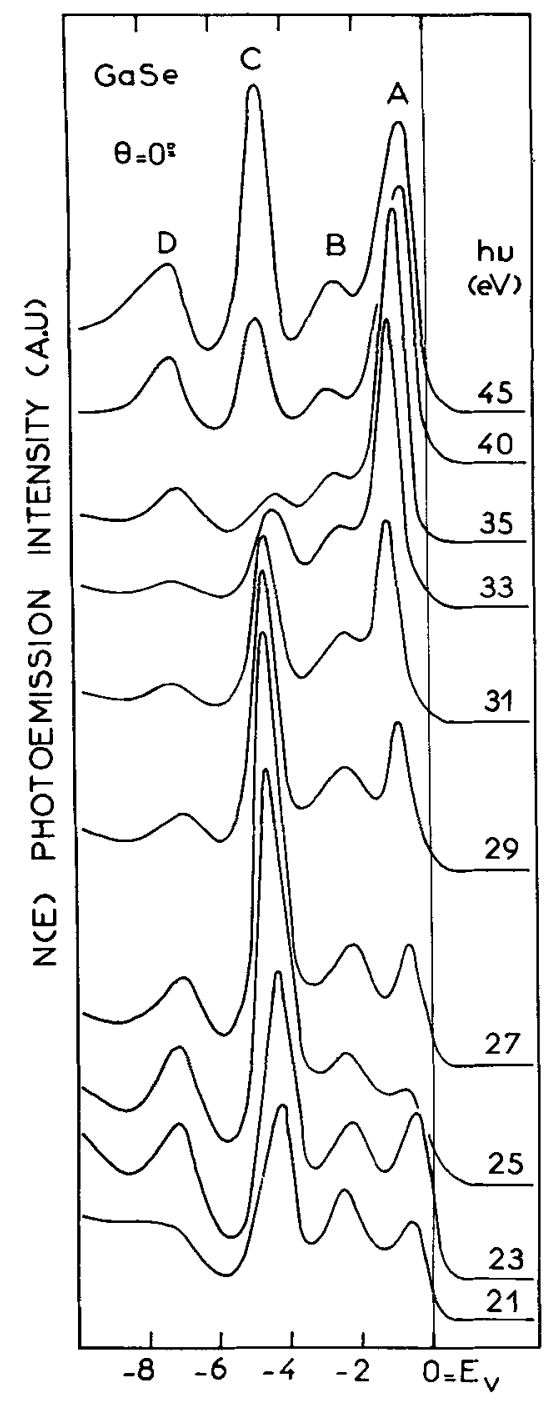

FIG. 9. - Energy distribution curves of the photoelectrons taken at $\theta=0$ for various photon energy $\hbar \omega$ between 21 and $45 \mathrm{eV}$.
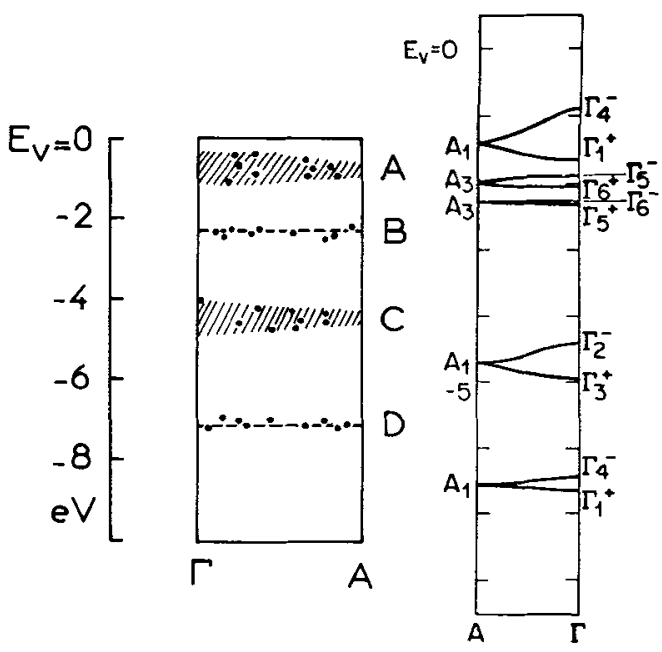

FIG. 10. - a) Energy dispersion of the bands in the direction $\Gamma \mathrm{A}$. b) Band structure calculated by M. Schlüter [13]. is in a very good agreement. However we have to notice that the hypothesis of free electron-like conduction band is perhaps not quite correct for a layer compound.

The discussion until now has been limited to the positions of the peaks in the photoemission spectra. In fact there is more physics in the peak intensities but the interpretation is more complicated and as shown by Liebsch [18] needs calculations based on a L.E.E.D. type formalism. We should expect a large development of this field very soon.

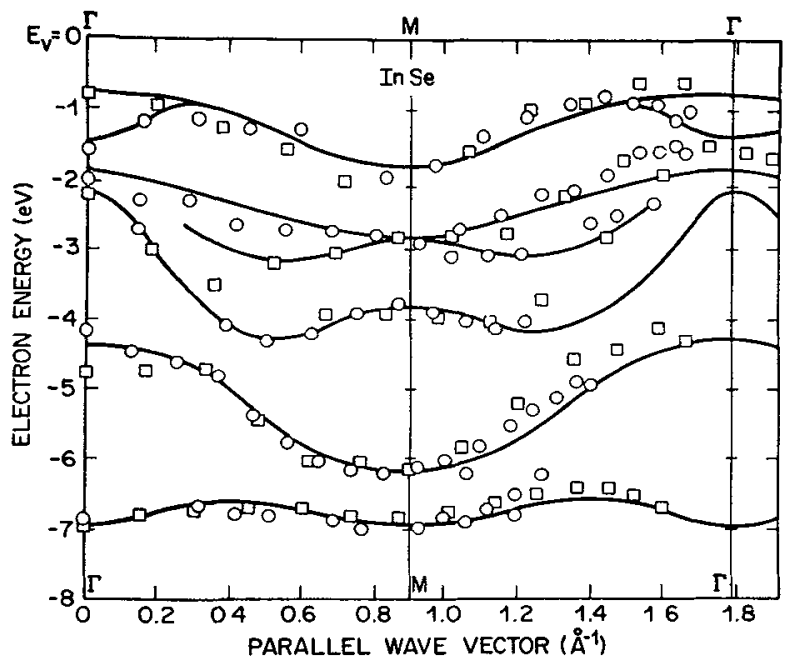

FIG. 11. - Two-dimensional band structure for InSe along the $\Gamma \mathrm{M} \Gamma$ direction. Open circles represent data for $\hbar \omega=18 \mathrm{eV}$; open squares represent data for $\hbar \omega=24 \mathrm{eV}$. Smooth curves have been drawn to pass near experimental points, as well as possessing mirror symmetry around the point $M$ (from Ref. [15]).

4. Three-dimensional semiconductors. - As I said in the beginning of this talk in three dimensional systems we have a lack of information about the normal component $k_{\perp}$. That explains why very few experiments have been reported [19] and why the interpretation of the results is not very satisfying.

$\mathrm{PbS}$.

Recently Granke, Ley and Cardona [20] have shown that the data on $\mathrm{PbS}$ (rocksalt structure) can be understood in terms of the one-dimensional density of states calculated along lines defined by $\mathbf{k}_{\|}=$constant in reciprocal space. They suppose that transition matrix elements are not strongly dependent on the energy of the initial state. The angular resolved photocurrent with electron energy $E$ created by photons of energy $\hbar \omega$ is :

$I(E) \sim \iiint \mathrm{d}^{3} \mathbf{k}_{\mathrm{v}} \delta\left(E_{\mathrm{v}}+E-\hbar \omega\right) \delta\left(\mathbf{k}_{\| \mathrm{v}}-\mathbf{k}_{\|}\right)$

where $\mathbf{k}_{\mathrm{v}}$ is the crystal momentum of a valence band states, $\mathbf{k}_{\|_{v}}$ the component of this momentum parallel to the surface, $\mathbf{k}_{\|}$the corresponding component of the photoelectron momentum and $E_{\mathrm{v}}$ the energy (measured 
with respect to the vacuum level) of the valence band state of momentum $\mathbf{k}_{\mathbf{v}}$.

Two modes of measurement are possible. The one used here corresponds to keep $\theta$ constant. However, theoretically it is more convenient to treat the case in which $\mathbf{k}_{\|}$is kept constant (varying $\theta$ while $E$ is being swept). Since for a given $\theta$ and $E, k_{\|}$is fixed only those valence electrons with $\mathbf{k}_{\| v}=\mathbf{k}_{\|}$contribute to the photo current. According to [6] the ARPES spectrum represents the density of states of the one-dimensional sections of the valence bands-obtained by setting

$$
\begin{gathered}
\mathbf{k}_{\| v}=\mathbf{k}_{\|} \\
I \sim \sum_{\text {val. band }}\left[\frac{\mathrm{d} E_{\mathrm{v}}}{\mathrm{d} k_{\perp \mathrm{v}}}\left(\mathbf{k}_{\| \mathbf{v}}, \mathbf{k}_{\perp \mathrm{v}}\right)\right]_{E_{\mathrm{v}}=h \omega-E_{\mathrm{c}}}^{-1}
\end{gathered}
$$

In view of the sharp peaks associated with the singularities in one-dimensional density of states, they estimated that the condition $\theta=$ constant (used in the experiments) did not significantly shift the peaks in an ARPES spectrum.

The figure 12 shows the Brillouin zone of a f.c.c. crystal. One dimensional densities of states were calculated along $k_{\| v}=$ constant, as indicated by the vertical lines using the Empirical Pseudopotential Method and the coefficients given by Kohn [21] et al. (the spin-orbit interaction is neglected).

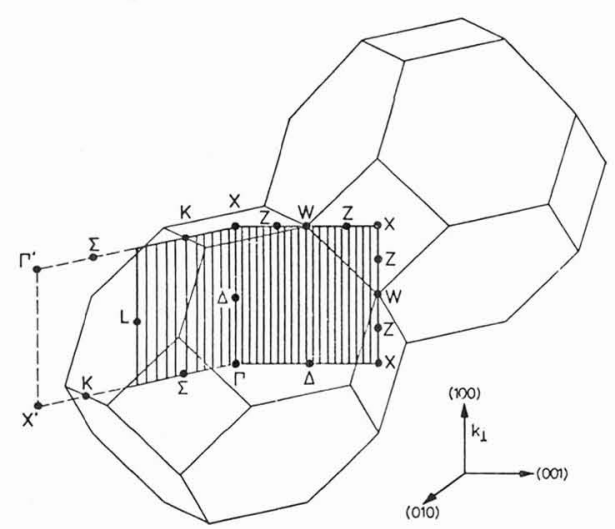

FIG. 12. - Extended zone scheme of the fcc-structure. The measurements were confined to irreducible wave vectors $\mathbf{k}_{\mathrm{v}}$ lying within the square $\mathrm{X}-\mathrm{X}-\mathrm{X}-\Gamma$. One dimensional densities of states were calculated along $\mathbf{k}_{\| \mathbf{v}}=$ constant, as indicated by the vertical lines. (Ref. [20].)

The energy dependence in $I(E)$, given by equation (7) as a function of $\mathbf{k}_{\| \mathbf{v}}$, is plotted in figure $13 a$ for the upper valence bands, mainly derived from the $3 p$ levels of sulphur. Full lines correspond to critical points located at $k_{\perp \mathrm{v}}=0$ (i.e. along $\Gamma-\Delta-\mathrm{X}$ ), dashed lines to critical points at $k_{\perp v}=2 \pi / a$ (i.e. along X-Z-W-Z-X), and dotted lines to critical points at some intermediate value of $k_{\perp v}$.

The figure $13 b$ shows the experimental peak positions versus the corresponding electron momentum component $\mathbf{k}_{\|}$(obtained for $\hbar \omega=21.2 \mathrm{eV}$ ). The crosses stand for intense peaks while the open circles
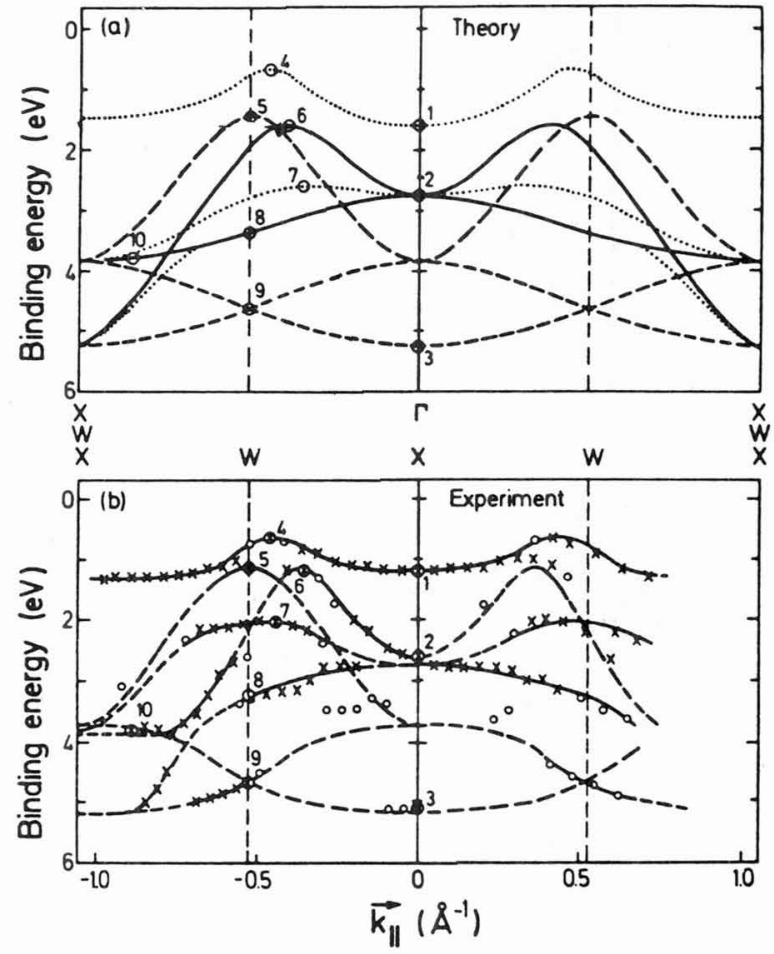

FIG. 13. - a) Dependence of the energies of critical points in the one-dimensional density of states on $\mathbf{k}_{\| \mathbf{v}}$. The solid lines refer to critical points at $k_{\perp \mathrm{v}}=0$, the dashed lines to critical points at $k_{\perp v}=2 \pi / a$ and the dotted lines to critical points at an intermediate value of $k_{\perp v}$. (From Ref. [20].) b) Experimental peak position versus electron momentum component $\mathbf{k}_{\|}$. The experimental points are connected by lines to give energy vs. momentum similar in shape to those of figure $13 a$.

refer to weak peaks or shoulders. It is possible to connect these discrete points by continuous lines very similar in shapes to those of figure $13 a$. Thereby we are able to assign every observed peak to a critical point in the one-dimensional density of initial states.

The agreement between theory and experiment is within what can be expected for the accuracy of any band structure calculation. Some minor disagreements are visible around $\left|\mathbf{k}_{\|}\right|= \pm 0.25(\AA)^{-1}$ where weak shoulders appear that do not correspond to any calculated critical point. The absence of about $30 \%$ of the predicted peaks is attributed to the absence of appropriate final state.

PbSe.

We have also performed experiments on crystals of the same family : PbSe. We have chosen to work at $\hbar \omega=35 \mathrm{eV}$. At this photon energy we expect that the mean free path of the photoelectron is of the same order of magnitude that the interatomic distance which means that we avoid the direct transitions band structure regime [2]. The experimental configuration used is shown on figure 14 . The figure 15 gives some of the photoemission spectra obtained at $\hbar \omega=35 \mathrm{eV}$ for different $\theta$. From these results it is possible to plot the peaks position versus $\mathbf{k}_{\|}$(Fig. 16). The three first valence band (within 0 and $-5 \mathrm{eV}$ ) are $\mathrm{p}$ derived, the 


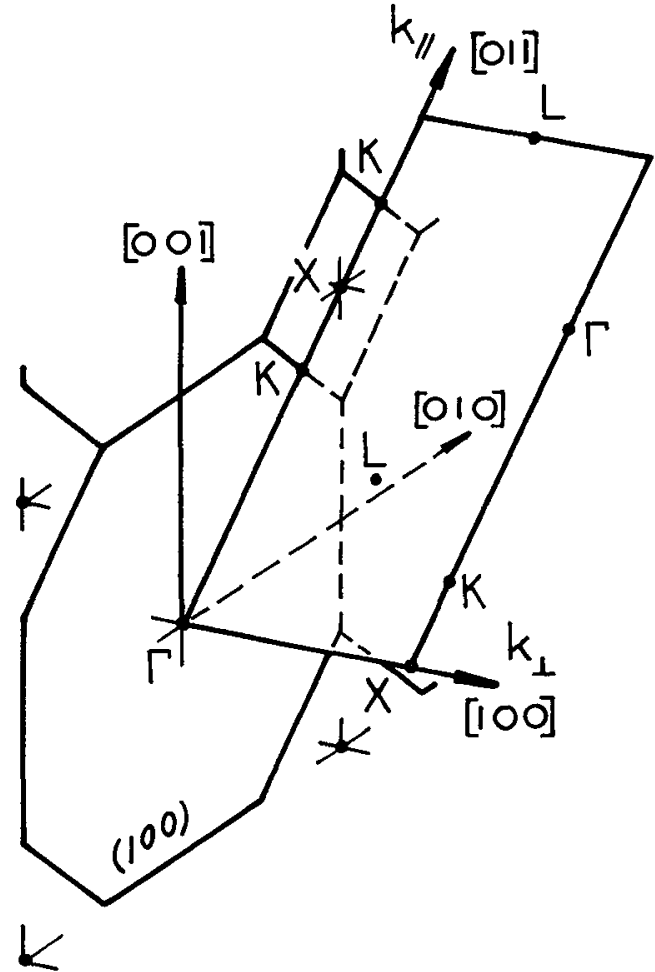

FIG. 14. - Extended zone scheme of PbSe; in our experimental configuration $k_{\|}$is along (011) and $k_{\perp}$ along (100).

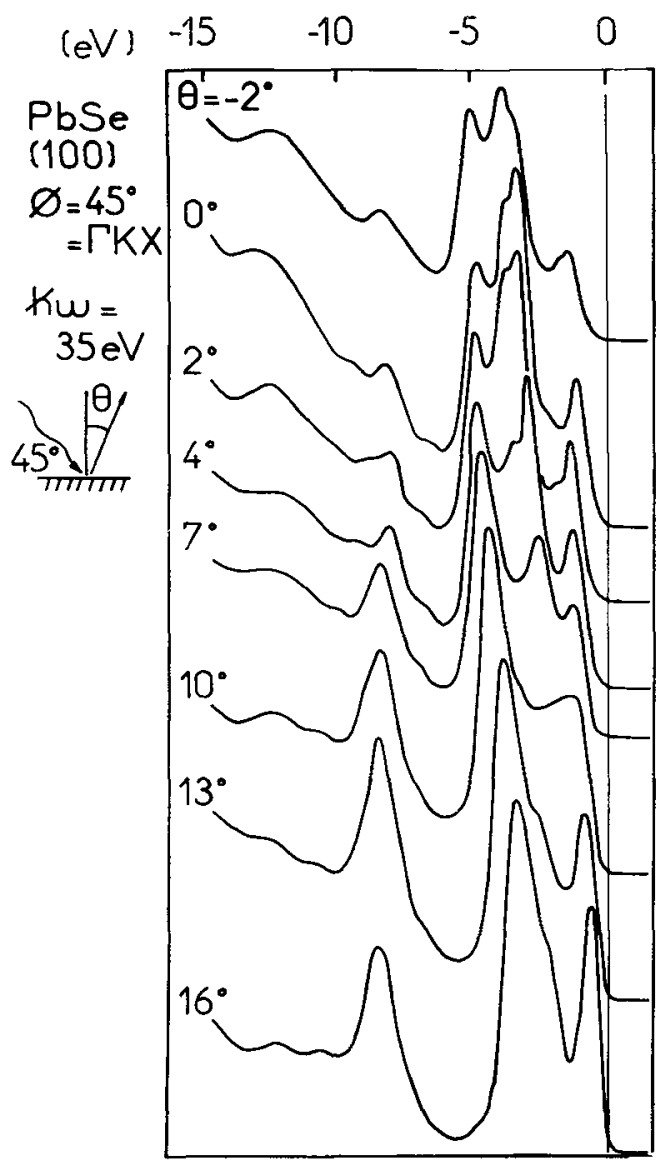

Frg. 15. - Angle resolved photoemission spectra at $\hbar \omega=35 \mathrm{eV}$ for $\theta$ varying between $-2^{\circ}$ and $16^{\circ}$ (but data have been taken each $3^{\circ}$ until $\theta=45^{\circ}$ ).

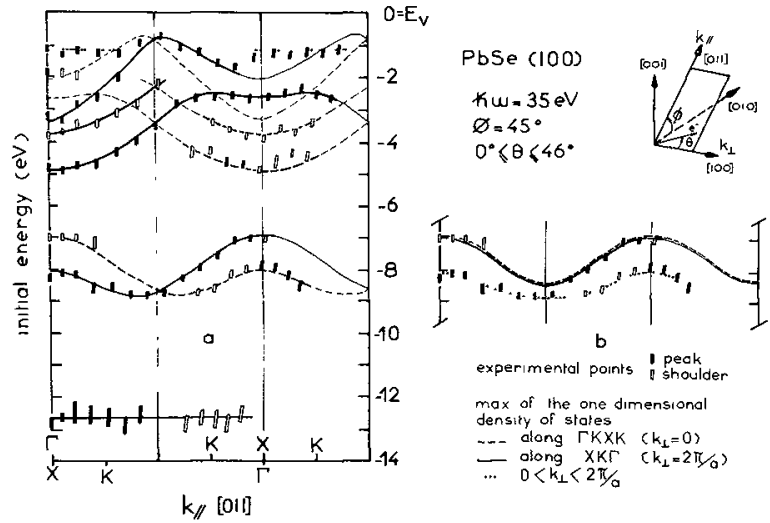

FIG. 16. - Experimental peak positions versus $k_{\|}$. stand for peaks, $\square$ for shoulders. The points are connected by lines to give curves similar in shape to those of figure 17.a) and $b$ ) correspond to two possible solutions for the first s band (mostly $6 \mathrm{~s}$ of $\mathrm{Pb}$ and $4 \mathrm{~s}$ of Se).

last two mostly s. For the last one ( $4 \mathrm{~s}$ of $\mathrm{Se})$ the cross section is low in this photon energy range : that explains the larger error bar.

We have represented on figure 17 the energy dependence of the maxima in $I(E)$ given by equation $(8)$ as a function of $\mathbf{k}_{\|}$. For that we have calculated the onedimensional density of states along $\mathbf{k}_{\perp}$ using the band

PbSe (100)

$\varnothing=45^{\circ}$

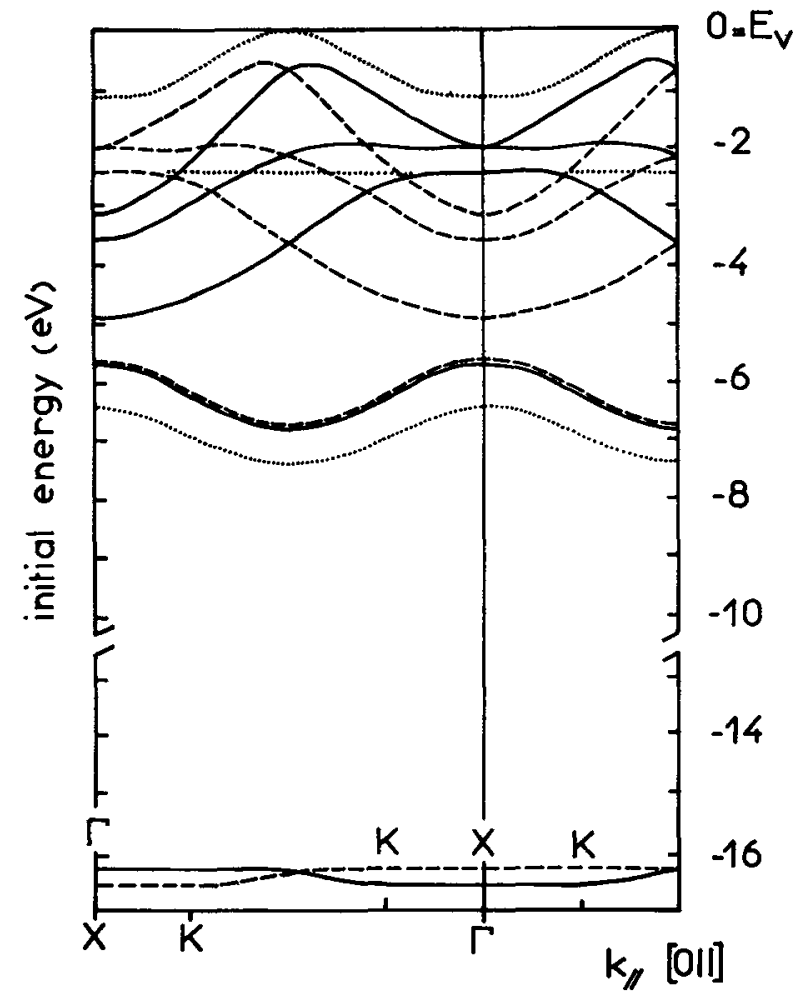

FiG. 17. - Variation of the maxima of the one-dimensional density of states given by equation (8) with $k_{\|}$. The solid lines refer to maxima along $\operatorname{XK} \Gamma\left(k_{\perp}=2 \pi / a\right)$, the dashed lines to maxima along $\Gamma \mathrm{KX} \Gamma\left(k_{\perp}=0\right)$, the dotted lines to maxima at intermediate value of $k_{1}$ (calculated with the band structure of Ref. [21]). 
structure of Kohn et al. [21]. The solid lines refer to maxima along $\mathrm{XK} \Gamma\left(k_{\perp}=2 \pi / a\right)$, the dashed lines to maxima along, $\Gamma \mathrm{KX} \Gamma\left(k_{\perp}=0\right)$, the dotted lines to maxima at intermediate value of $k_{\perp}$. We have drawn similar lines between the experimental points. Excepted the structure at $-1.2 \mathrm{eV}$, all the others take their origin along $\Gamma \mathrm{KXK}\left(k_{\perp}=0\right)$ and $\mathrm{XK} \Gamma\left(k_{\perp}=2 \pi / a\right)$; $a)$ and $b$ ) correspond to two possible solutions for the fourth valence band.

In fact if is easy to show [22] that for high symmetry directions, the lines of figure 17 are just the band structure. Following that we have represented on figure 18 the experimental band structure along $\Gamma \mathrm{KX}$.

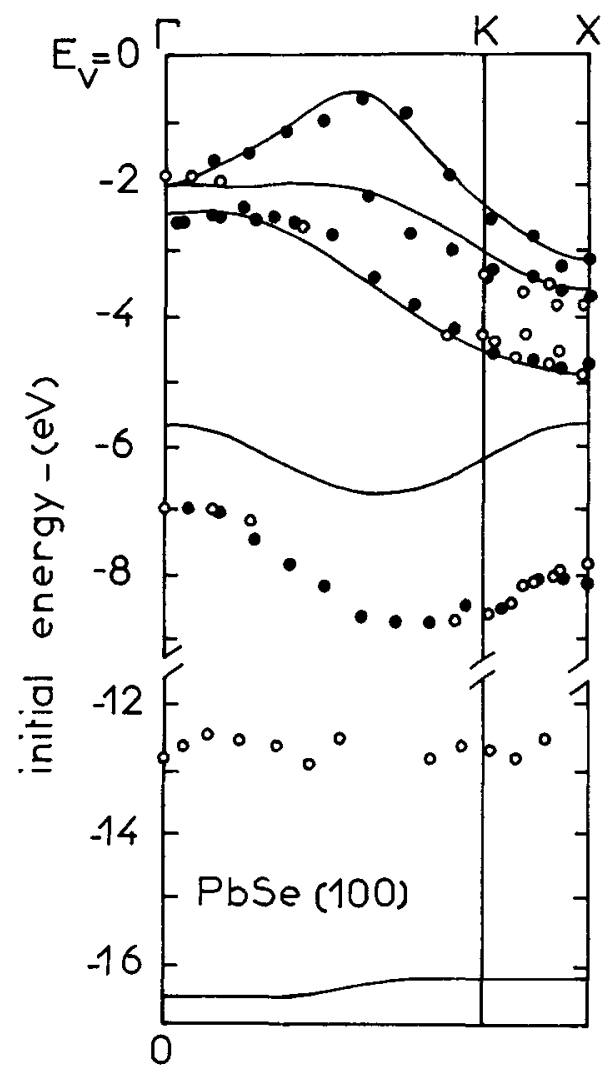

FIG. 18. - Band structure of PbSe. The solid lines are the bands calculated by Kohn et al. (Ref. [21]); $O$ and the experimental points.
The full lines correspond to the calculation of Kohn et al. [21]: the experimental points come from $\Gamma \mathrm{KXK}$ and $O$ from XK $\Gamma$.

The agreement for the $\mathrm{p}$ derived bands is remarquable. As already shown by X.P.S. [23] measurements the positions of the calculated $s$ derived bands are incorrect.

In table I we compare the experimental and calculated energies $[21,24,25]$ of some critical points and of the spin-orbit splitting at $\Gamma$ and $X$.

Finally we have performed photoemission experiments at the normal $\left(\theta=0\right.$ and $\left.k_{\|}=0\right)$. In a direct

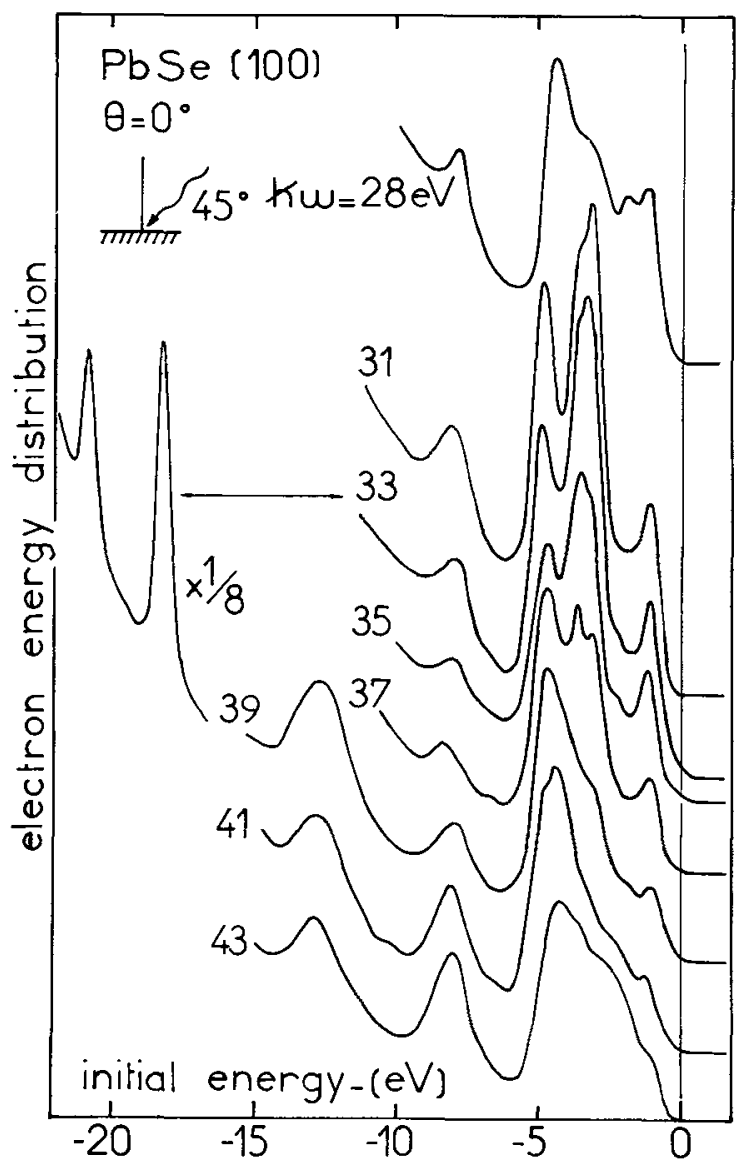

FIG. 19. - Photoemission spectra taken at normal emission $(\theta=0$, $k_{\|}=0$ ) for photon energy $\hbar \omega$ between 28 and $43 \mathrm{eV}$.

TABLE I

Energ. position of the electronic levels of the valence band of $\mathrm{PbSe}$ (in $\mathrm{eV}$ )

\begin{tabular}{|c|c|c|c|c|c|}
\hline \multirow[b]{2}{*}{ Band } & \multirow[b]{2}{*}{ Sym. } & \multirow[b]{2}{*}{ Exp. } & \multicolumn{3}{|c|}{ Theory } \\
\hline & & & $x$ & $\hat{\beta}$ & \\
\hline- & - & - & - & - & - \\
\hline 5 & $\Gamma_{8}^{-}$ & -2 & -1.9 & -2 & -2 \\
\hline 4 & $\Gamma_{8}^{-}$ & & & & \\
\hline 3 & $\Gamma_{6}^{-}$ & -2.6 & -2.5 & -2.55 & -2.6 \\
\hline 2 & $\Gamma_{6}^{+}$ & -7 & -5.2 & $-\quad 5.7$ & -6.1 \\
\hline 1 & $\Gamma_{6}^{+}$ & -127 & -14.9 & -16.4 & -14.4 \\
\hline$\Delta_{\mathrm{s}-0}$ & & 0.6 & 0.6 & 0.55 & 0.6 \\
\hline
\end{tabular}

x) From reference [24].

B) From reference [21]

i) From reference [25].

$\Delta_{s-0}$ is the spin-orbit. 
transitions model we should be able to map the bands along $\Gamma \mathrm{X} \Gamma$; in fact as shown on figure 19 , no dispersion is observed. This is coherent with the previous results showing that at these photon energies one observes the density of states.

5. Metals. - I have chosen to discuss only two metals : copper and nickel. The reason of this choice is that they have $\mathrm{d}$ bands and very similar electronic structure. However their photoemission spectra look quite different : the experimental peaks of copper are very sharp, compared to nickel. To explain this difference the following reasons have been given :

- Lifetime of the hole [26] ; copper has a very long lifetime for the hole created in the d band (low density of states near the Fermi level). This gives rise to photoemission spectra with well defined structure. On the contrary Ni has a very high density of states and a short hole lifetime. A hole created near the bottom of the $d$ band will decay by Auger processes before it has the possibility to move on an other atom. Pendry [26] has introduced a lifetime which is a strong function of $\left(E-E_{\mathrm{f}}\right)$ and improved the agreement between theory and experiment.

- Correlations; Friedel [27] has suggested an other mechanism. In the case of nickel, correlations between

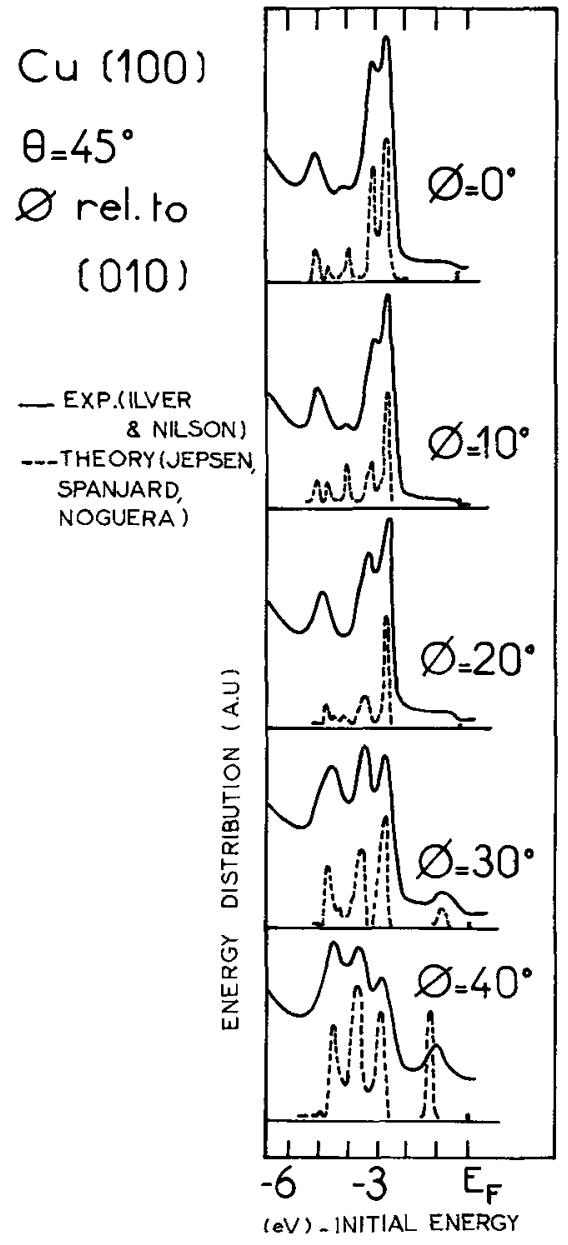

Fig. 20. - Theoretical (..) and experimental (-) photoemission spectra of $\mathrm{Cu}(100)$. (Ref. [27].) electrons can give rise to a slightly random potential and $\mathbf{k}$ is no longer exactly a good quantum number. To study this effect Noguera et al. [27] (this conference) have used a crude model (Hubbard with one $s$ band) and have shown that this effect should be maximum in the half of the $d$ band. We have to notice that it is not easy to know the importance of this effect.

Cu.

It seems that copper is an ideal metal for photoemission : by ideal I mean that the agreement between numerous experiments and theory is remarkable. Different angular [26], [27] resolved photoemission calculations have been performed. All of them use the same formalism (the final state is the complex conjugate of the L.E.E.D. wave function of an electron coming from the direction of the detector, muffin-tin potentials...); the main difference is in the shape of the surface potential.

The results of Spanjard et al. [27] are shown on figure 20 for $\mathrm{Cu}(100)$ (dashed line); the full line corresponds to the experiment of Ilver and Nilson [28]. One can see that remarkable agreement is obtained for different azimuthal angles (not only for the positions of the peaks but also for the intensity). Similar results are observed on the face (111) (Fig. 21). An important

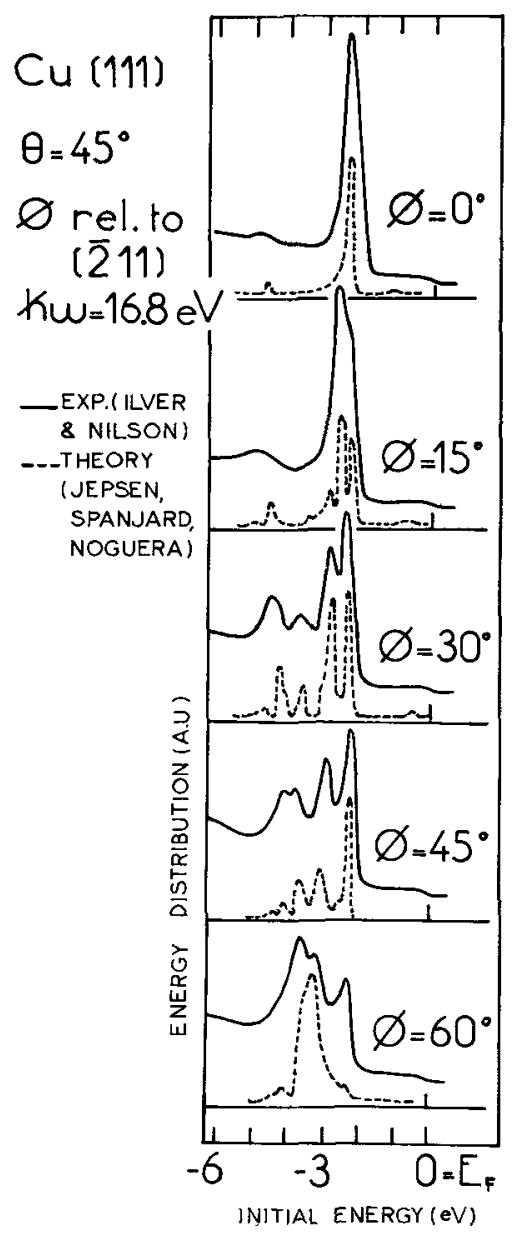

FIG. 21. - Theoretical (- - ) and experimental (-) photoemission spectra of $\mathrm{Cu}$ (111). (Ref. [27].) 
point is that, at $\hbar \omega=16.8 \mathrm{eV}$, nearly all the observed experimental peaks are due to direct transitions in which both $k_{\|}$and $k_{\perp}$ are conserved.

Recently Wehmer et al. [29] have used angular resolved photoemission at the normal $\left(\theta=0, k_{\|}=0\right)$ over a large energy range $(30 \leqslant \hbar \omega \leqslant 160 \mathrm{eV})$ to map the bulk band structure (Fig. 22). The experimental peak positions are compared with the Burdick's band structure [30]. The experimental points are derived from the equation (5) by the following steps. From the measured peak positions, relative to the Fermi level, it is easy to calculate the final state energy. The magnitude of the final state wave vector is given by

$$
|\mathbf{K}|=\left(\frac{2 m E_{\mathrm{f}}}{\hbar^{2}}\right)^{1 / 2}
$$

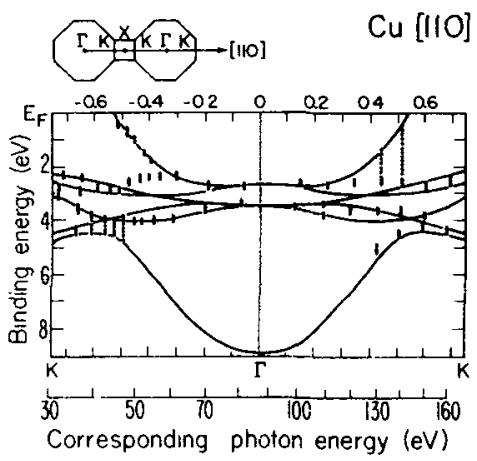

Fig. 22. - Comparison of experimental peak position with Burdick's band structure of $\mathrm{Cu}$ for the direction (110). The bottom inset establishes a correspondence between photon energy and the part of the Brillouin sampled (Ref. [29]).

where the zero of the free electron scale is taken to the bottom of the free electron-like bands in Burdick's band structure. The relation $\mathbf{K}_{\perp}=\mathbf{k}_{\perp}+\mathbf{G}_{\perp}$ yield $\mathbf{k}$ in the first Brillouin zone and the corresponding values are plotted as bars in the figure. The inset at the bottom of the panel establishes a correspondence between $\hbar \omega$ and the part of the B.Z. sampled. The more interesting point is the pronounced s-p resonance at $\hbar \omega=45 \mathrm{eV}$ for bulk transitions arising near $k=(-0.5,-0.5,0)$ in the B.Z. It is quite clear that we obtain the dispersion of the $s$ band. However we have to point out that above $70 \mathrm{eV}$, the mean free path of the photoelectron is quite short $(\sim 3 \AA)$ and that should break the coherence of the wave (non-conservation of $k_{\perp}$ ). Above this energy we are in a X.P.S. regime as shown by Feibelman and Eastman and we should obtain the density of states along (110). Also this direct transitions model does not explain completely the results of Heimann et al. [31] obtained at lower photon energies $(\hbar \omega=16.8$ and $21.2 \mathrm{eV})$.

Ni.

I would like to discuss now a resonance effect observed on one of the structures of the valence band of $\mathrm{Ni}$. We have observed it in an angle resolved photoemission experiment but it should be visible in angle integrated experiments also [32].
X-ray (X.P.S.) and ultraviolet (U.P.S.) photoemission spectra $[33,34,35]$ of a clean Ni $(100)$ surface show a weak structure at approximately $6 \mathrm{eV}$ below the Fermi level. The interpretation of this feature has been quite controversial. The X.P.S. core-level spectra $(2 s, 3 s, 3 p)$ have also a satellite structure at about the same energy, which has been attributed to a localized many body effect in the final state [36]. It has been suggested [34] that the X.P.S. valence-band feature might be analogous to this because of the strongly localized character of the d-bands in nickel.

However, in recent experiments with synchrotron radiation between $23 \mathrm{eV}$ and $40 \mathrm{eV}$ Smith et al. [35] have concluded that this weak structure is consistent with a simple band picture for an electronic transition originating from the $\mathrm{s}-\mathrm{p}$ band, $\Delta_{1}$.

We have studied this structure experimentally in photoemission from $\mathrm{Ni}$ for photon energies $\hbar \omega$, ranging from $40 \mathrm{eV}$ to $100 \mathrm{eV}$. We observe a strong resonance enhancement for $\hbar \omega$ in the neighbourhood of the 3p energy levels $\left(E_{\mathrm{p}^{3 / 2}}=66 \mathrm{eV} ; E_{\mathrm{p}^{1 / 2}}=67.6 \mathrm{eV}\right)$. Off resonance the structure is still visible, both on the high- and low-energy side, but with rapidly falling intensity, especially on the low-energy side. The asymmetric line shape [37] is suggestive of resonant interaction between an excited quasi-discrete level $\left(3 p^{5} 3 d^{10} 4 s\right)$ and an ionized continuum $\left(3 p^{6} 3 d^{8} 4 s\right.$ plus emitted electron) : this is the model we choosed to interpret our data.

The experiments, which are not discussed here in detail, were performed at the Orsay ACO storage ring. Throughout the experiment, outgoing electrons were collected normal to the nickel single crystal (100) surface. This was prepared by the usual methods : ion bombardment and annealing under ultra-high vacuum (less than $2 \times 10^{-10}$ torr). The surface was monitored by low-energy electron diffraction and Auger spectroscopy and care was taken to avoid oxygen contamination, since the oxygen $2 \mathrm{p}$ orbitals give by themselves a peak at about $6 \mathrm{eV}$ below the nickel Fermi level.

Experimental results are shown in figure 23 for twelve different incident photon energies, between $\hbar \omega=63 \mathrm{eV}$ and $\hbar \omega=83 \mathrm{eV}$. For $\hbar \omega=63 \mathrm{eV}$ the photoemission spectrum consists of two main features : a sharp peak structure $A$ at about $1.5 \mathrm{eV}$ below the Fermi level, due to the $3 \mathrm{~d}$ bands of nickel, and the much weaker structure $\mathrm{B}$ at $6 \mathrm{eV}$ below $E_{\mathrm{F}}$. The structure $\mathbf{B}$ shows strong resonant behaviour as $\hbar \omega$ is tuned through the energy corresponding to the $3 \mathrm{p}$-to-conduction-band transition (see $\hbar \omega=67 \mathrm{eV}$ curve in figure 23). On the high-energy side of $\mathrm{B}$, for $\hbar \omega \geqslant 69 \mathrm{eV}$, a new peak appears : this is a wellcharacterized Auger transition and it is indicated by an arrow on figure 23.

The area under the structure B as a function of $\hbar \omega$, i.e. the line shape of the experimental resonance curve, is plotted in figure 24 . It shows a marked asymmetry 


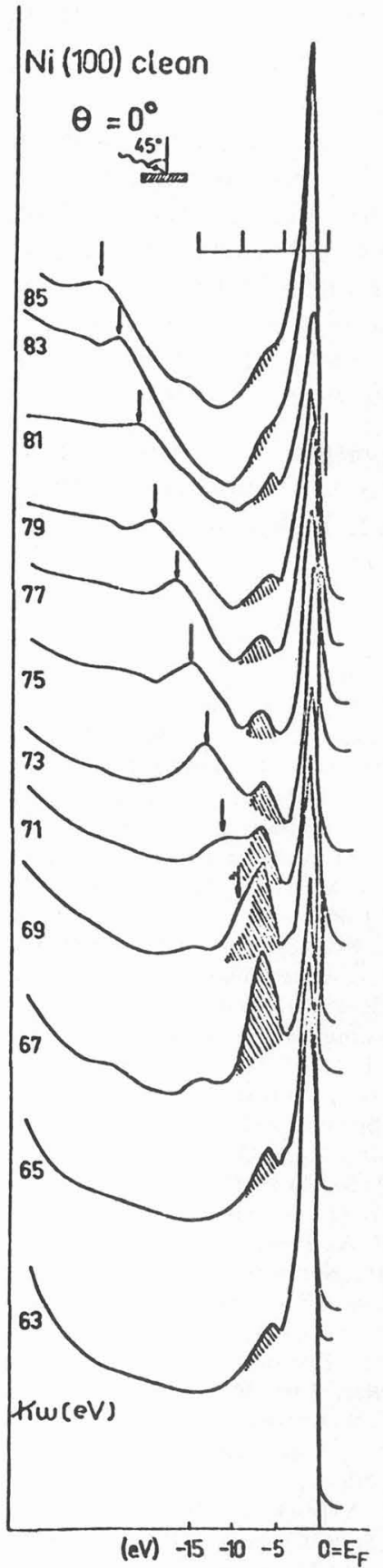

FIG. 23. - Photoemission spectra of Ni $(100)$ for photon energy $\hbar \omega$ between 63 and $81 \mathrm{eV}$. The peak $\mathrm{A}$ corresponds to the $\mathrm{d}$ bands; $B$ (dashed area) is the structure located at $6 \mathrm{eV}$ from the Fermi level. The arrows indicate the Auger transition.

which leads naturally to an interpretation of the phenomenon as a Fano resonance [37].

Considered from the atomic point of view, each $\mathrm{Ni}$ atom in the metallic ground state of nickel can be thought of as being, from the electronic point of view, in a coherent superposition of various configurations, but mainly of $(3 \mathrm{~d})^{9}(4 \mathrm{~s})$ and $(3 \mathrm{~d})^{10}$. It is the former which is responsible for the effect we find. The absorption process

$$
(3 p)^{6}(3 d)^{9} 4 s+\hbar \omega \rightarrow(3 p)^{5}(3 d)^{10} 4 s
$$

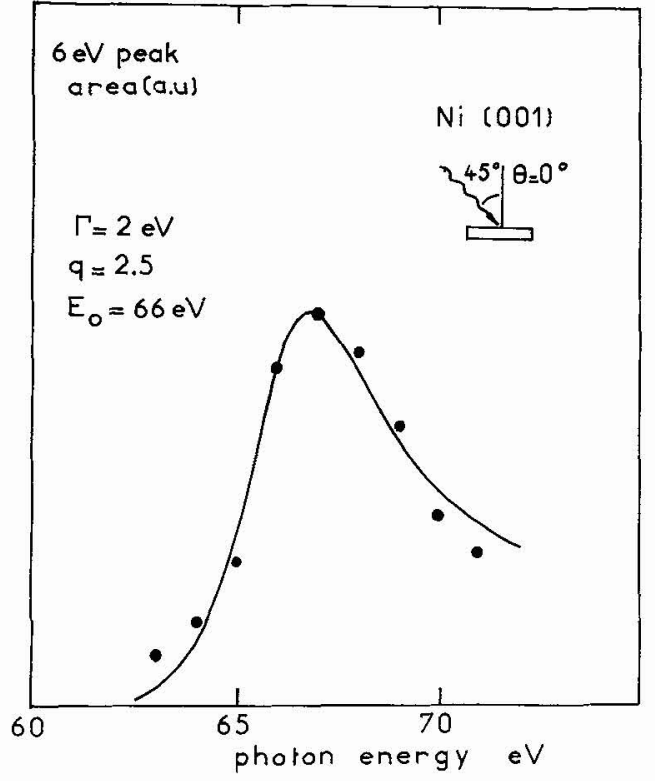

Fig̣. 24. - Plots of the area of the B peak structure versus the photon energy in the vicinity of the $3 p$ levels. The points are experimental. The full line corresponds to the values given by equations (9)-(12) with the following parameters $\Gamma=2 \mathrm{eV}, q=2.5$ and $E_{0}=66 \mathrm{eV}$.

produces a quasi-discrete state which, through a Coulomb (autoionizing) interaction $V_{E}$, is hybridized with a continuum

$(3 p)^{5}(3 d)^{10} 4 s \leftrightarrow(3 p)^{6}(3 d)^{8} 4 s+$ unbound electron .

This Fano-type interaction, [37] with its characteristic asymmetric lineshape, has been suggested to be the physical origin of the structure observed in softX-ray absorption [38, 39] and in electron-loss spectra [40] for nickel metal. The Fano interaction yields a spectral line with its typical resonance-antiresonance features and described by the simple formula

$$
I=\frac{(q+\varepsilon)^{2}}{1+\varepsilon^{2}}
$$

where

$$
\begin{aligned}
\varepsilon & =\frac{\left(\hbar \omega-E_{0}\right)}{\Gamma} \\
\Gamma & =\pi\left|V_{E}\right|^{2}
\end{aligned}
$$

is the spectral half-width of the autoionized state, and

$$
q=\frac{(3 \mathrm{p}|r| 3 \mathrm{~d})}{(3 \mathrm{~d}|r| \varepsilon \mathrm{f})} \times \frac{1}{\pi V_{E}} .
$$

In these formulae $E_{0}$ is the displaced energy for the transition $r$ indicates dipole matrix elements and $\mid \varepsilon f)$ is the f-partial wave of the unbound electron $[40,41]$.

We have fitted our experimental data to equation (9) and, as shown in figure 24, we find good agreement for $q=2.5, \Gamma=2.0 \mathrm{eV}$ and $E_{0}=66 \mathrm{eV}$. Our value for $q$ is considerably larger than those obtained from either energy loss spectra $[40,42,43](q=0.9$ and $q=1.3)$ 
or theoretical calculations $[40,44](q=1.8)$. The values of $\Gamma$ and $E_{0}$ are in excellent agreement with previous values $[40,44]$. It should be emphasized that because of the experimental resolution and uncertainties, and the atomic model and simplifying assumptions [41], a fit better than the one obtained and quoted above can only be considered fortuitous. However, as shown in reference [32], the metallic character cannot be completely ignored in our interpretation.

It should be of considerable interest to study the photoelectron spectrum of atomic $\mathrm{Ni}$ in the same range of energies. A comparison with our present experiments would then yield information on the relative contributions of the one-electron band struc- ture and of the electron-electron atomic-like correlations in metallic $\mathrm{Ni}$.

6. Conclusion. - In my talk I have reviewed only energy distribution experiments : with the possibilities of source as synchrotron radiation (tunability, high flux, polarization, higher energy available...) it is easy to understand why this field is growing so fast.

The last parameter that we have to measure now is the spin of the photoelectron; I bet that, at the next Vacuum Ultraviolet Radiation Physics Conference this will be done by many teams.

Acknowledgments. - I would like to thank J. Lecante, P. Thiry, C. Noguera, D. Spanjard, R. Pinchaux and Pr. L. Falicov for very helpful discussions.

\section{References}

[1] Gobeli, G. W., Allen, F. G., Kane, E. O., Phys. Rev. Lett 12 (1964) 94.

Kane, E. O., Phys. Rev. Lett. 12 (1964) 97.

[2] Feibelman, P. J. and Eastman, D. E., Phys. Rev. B 10 (1974) 4932.

[3] Lepere, D., Nouv. Rev. Opt. 6 (1975) 173 and

Petroff, Y., Thiry, P., Pinchaux, R., Lepere, D., 5th International Conference on Vacuum Ultraviolet Radiation Physics, Montpellier (September 1977).

[4] Lloy, D. R., Quinn, C. M., Richardson, N. V. and Williams, P. M., Commun. Phys. 1 (1976) 11

[5] Larsen, P. K., Margaritondo, G., Rowe. J. E., Schluter, M. and SMith, N. V., Phys. Leti. 58A (1976) 423.

[6] Thiry, P., Petroff, Y., Pinchaux, R., Ballu, Y., Guillot, C., LeCANTE, J., Layered Semiconductors and Metals. Satellite Conference of I.C.P.S. Bari, Sept. 76 (unpublished) and Solid State Commun. 22 (1977) 685

[7] Megovern, I. T., Parke, A. and Williams, R. H., J. Phys. C 9 (1976) L-511.

[8] Smith. N. V., Traum, M. M. and Di Salvo, F. J., Solid State Commun. 15 (1974) 211.

Smith, N. V., Traum, M. M., Phys. Rev. B 11 (1975) 2087.

[9] SChluter, M., Nuovo Cimento 13B (1973) 313.

[10] Rowe, J. E., Margaritondo, G., Kasper, H. and BaldeRESCHI, A., Solid State Commun. 20 (1976) 921.

[11] KowalczyK, S. P., Ley, L., McFeely, F. R. and Shirley, D. A., Solid State Commun. 17 (1975) 463.

[12] ThtR Y, P. (unpublished)

[13] Schluter, M., Camassel, J., Kohn, S., Voltchovsky, J. P., Shen, Y. R. and Cohen, M. L., Phys. Rev. B 13 (1976) 3534 and

SCHLUTER, M., private communication.

[14] Excepted around $r$ for the structures $A$ and $C$ which correspond to bands having a three-dimensional character.

[15] Larsen. P. K., Chiang. S. and Smith. N V., Phys Rev B 15 (1977) 3200.

[16] GrandKe, T. and Ley, L. (this conference).

[17] LaRsen. P. K., Schluter, M. and SMith, N. V., Solid State Commun. 21 (1977) 775.

[18] LiebsCH, A., Solid State Commun. 19 (1976) 1193.

[19] Smith, N. V. and Traum, M M.. Phys Rev. Lett. 31 (1973) 1247:

Williams, P. M., Butcher, P., Wood, J. and JAcobi, K., Phys. Rev. B 14 (1976) 3215.

[20] Grandke, T., Ley, L. and Cardona, H., Phys. Rev. Lett. 38 (1977) 1033
[21] Kohn, S. E., Yu, P. Y., Petroff, Y., Shen, Y. R., Tsang, Y. and Cohen, M. L., Phys. Rev. B 8 (1973) 1477.

[22] Thiry, P., Pinchaux. R., Martinez, G., Petroff, Y., Lecante, J., Paigne, J., Ballu, Y., Guillor, P. (to be published).

[23] McFeely, F. R., KowalczyK, S., Ley, L., Pollak, R. A. and SHIRley, D. A., Phys. Rev. B 7 (1973) 5228.

[24] Martinez, G., Schluter, M. and Cohen, M. L., Phys. Rev. B 11 (1975) 660.

[25] Herman, F., Kortum, R. L., Ortendurger, I. and Vandyke, J. P., J. Physique Colloq. 29 (1968) C4-62.

[26] Pendry, J. B. and Hopkinson, J. F. L., Int. Conf. on Physics of Transition Metals, Toronto (15-19 August 1977);

PendRY, J. B., Surf. Sci. 57 (1976) 679.

[27] Jepsen, D. W., SPanjaArd, D., Noguera, C., this conference and to be published.

[28] Liver, L., Nirsson, P. O., Solid State Commun. 18 (1976) 677.

[29] Wehmer, P. S., Apal, G., Williams, R. S., Stohr, J. and ShiRLeY, D. A. (this conference).

[30] Burdick. G. A., Phys. Rev. 129 (1963) 138.

[31] Heimann, P., Neddermeyer, H. and Roloff, H. F., Phys. Rev. Lett. 37 (1976) 775.

[32] Guillor, C., Ballu, Y., Paigne, J., Lecante, J., Jain, K. P., Thiry, P., Pinchaux, R., Petroff, Y., Falicon, L. M., Phys. Rev. Lett. 39 (1977) 1632

[33] Hufner, S., Wertheim, G. K., Phys. Lett. 51A (1975) 299

[34] Kemeny, P. C., Shevchik, N. J., Solid State Commun. 17 (1975) 255.

[35] Smith, R. J., Anderson, J., Hermanson, J. and Lapeyre, G. J., Solid. State Commun. 21 (1977) 459.

[36] Kotani, A. and Toyozawa, Y., J. Phys. Soc. Japan 35 (1973) 1073 and 37 (1974) 912.

[37] Fano, U., Phys. Rev. 124 (1961) 1866.

[38] Sonntag, B., Haensel, R. and Kunz, C., Solid State Commun. 7 (1969) 597.

[39] Brown, F. C., Gahwiller, C. and Kunz, A. B., Solid State Commun. 9 (1971) 487

[40] Dietz, R. E., McRae, E. G., Yafer, Y. and Caldwell, C. W., Phys. Rev. Lett. 33 (1974) 1372.

[41] A partial p-wave, which also contributes, gives a considerably smaller matrix element and can be neglected. See reference $[40]$.

[42] Davis, L. C. and Feldkamp, L. A., Solid State Commun. 19 (1976) 413.

[43] Colliex, C. and Trebila, P., Phys. Fenn. 9 Suppl. S1 (1974) 83.

[44] MCGuiRe, E. J., Sandia Laboratories Research Reports No. Sc-RR-710835 (unpublished), as quoted in reference [40]. 